\title{
Optically controlled magnetic-field etching on the nano-scale
}

\author{
Takashi Yatsui ${ }^{1}$, Toshiki Tsuboi ${ }^{1}$, Maiku Yamaguchi ${ }^{1}$, Katsuyuki Nobusada ${ }^{2}$, Satoshi Tojo ${ }^{3}$, Fabrice Stehlin ${ }^{4}$, \\ Olivier Soppera ${ }^{4}$ and Daniel Bloch ${ }^{5}$
}

Electric and magnetic fields play an important role in both chemical and physical reactions. However, since the coupling efficiency between magnetic fields and electrons is low in comparison with that between electric fields and electrons in the visible wavelength region, the magnetic field is negligible in photo-induced reactions. Here, we performed photo-etching of $\mathrm{ZrO}_{2}$ nano-stripe structures, and identified an etching-property polarisation dependence. Specifically, the etching rate and etched profiles depend on the structure width. To evaluate this polarisation-dependent etching, we performed numerical calculations using a finite-difference time-domain method. Remarkably, the numerical results revealed that the polarisation-dependent etching properties were determined by the magnetic field distributions, rather than the electric field distributions. As nano-scale structures induce a localised magnetic field, the discovery of this etching dependence on the magnetic field is expected to introduce a new perspective on advanced nano-scale structure fabrication. Light: Science \& Applications (2016) 5, e16054; doi:10.1038/Isa.2016.54; published online 25 March 2016

Keywords: nano-scale, near-field etching, optically controlled magnetic-field interaction

\section{INTRODUCTION}

Use of the near-field allows nano-scale imaging below the diffraction limit of light to be performed ${ }^{1}$. This technique has provided a means of visualising the point dipole in a single molecule ${ }^{2}$, centre-of-mass wavefunctions of an exciton in a quantum $\operatorname{dot}^{3}$, and molecular resolution imaging using pressure-assisted Raman imaging ${ }^{4}$.

Further, detailed study of electric fields in nano-scale materials has allowed the magnetic permeability in these substances to be tuned, while the recently developed nano-scale periodic-structure fabrication method ${ }^{5}$ has realised the predictions of Veselago ${ }^{6}$. In addition, the super-reflection property that originates from the negative refractive index has led to the realisation of super-resolution imaging and fabrication ${ }^{7}$. Many related applications have been developed, including optical cloaking ${ }^{8}$ and light harvesting devices ${ }^{9}$. However, although new phenomena have been observed in nano-scale materials as a result of adjustment of the magnetic permeability, the chemical and/or physical reactions within these substances are still determined by the electric field ${ }^{7}$. Further, the interaction of the magnetic field with the material excitation is considered to be negligible, because of the low coupling efficiency between magnetic fields and electrons.

In this study, nano-scale photo-etching of $\mathrm{ZrO}_{2}$ nano-stripe structures is performed, and the effects of the electric and magnetic fields on the etching properties are examined. Polarisation-dependent etching is observed, which is subsequently examined using a finite-difference time-domain (FDTD) approach.

\section{MATERIALS AND METHODS}

$\mathrm{ZrO}_{2}$ nano-stripe preparation

Polarisation-dependent nano-scale etching was performed on $\mathrm{ZrO}_{2}$ nano-stripe structures. The $\mathrm{ZrO}_{2}$ nano-stripe patterns were prepared using the deep-ultraviolet (DUV) interferometric lithography method reported in Ref. 10. This approach utilises a negative-tone resist based on photosensitive metal-oxo clusters, which are crosslinked and mineralised using DUV laser irradiation. Zirconium (IV) propoxide $\left(\mathrm{Zr}(\mathrm{OPr})_{4}\right)$ (Sigma-Aldrich) was used as a metal alkoxide, and methacrylic acid (MMA) (Sigma-Aldrich) was used as a ligand to form a metal complex, with a molar ratio of $\mathrm{Zr:MAA}=1: 8$. Water and isopropanol were added to adjust the physico-chemical properties of the resist. DUV irradiation led to the cross-linked structure to form $\mathrm{ZrO}_{2}$, after treatment of the non-exposed part with cyclohexanone ${ }^{11}$.

\section{Nano-scale etching}

$\mathrm{O}_{2}$ in atmosphere was used as a source gas, and radical oxygen was used to etch the $\mathrm{ZrO}_{2}{ }^{12}$. To dissociate the $\mathrm{O}_{2}$, we used a continuous-wave (CW) He-Cd laser $(\lambda=325 \mathrm{~nm} ; 3.81 \mathrm{eV}$; excitation power: $0.8 \mathrm{~W}$ $\left.\mathrm{cm}^{-2}\right)$. Thus, the incident photon energy was lower than the dissociation energy of the $\mathrm{O}_{2}(5.12 \mathrm{eV})^{13}$, and therefore, $\mathrm{O}_{2}$ dissociation occurred on the $\mathrm{ZrO}_{2}$ surface only. The absorption edge of a $\mathrm{ZrO}_{2}$ nano-stripe pattern is approximately $4.96 \mathrm{eV}(250 \mathrm{~nm})^{11}$; thus, we could exclude the effect of the carrier generation in the $\mathrm{ZrO}_{2}$ nanostripe patterns. To evaluate the resultant changes in the threedimensional $\mathrm{ZrO}_{2}$ structure, the surface structure was evaluated using

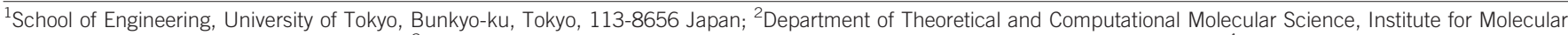

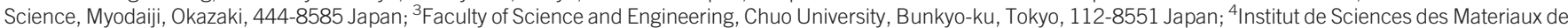

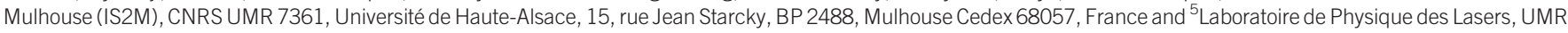
7538 du CNRS, Université Paris13-Sorbonne-Paris-Cité F-93430 Villetaneuse, France

Correspondence: T Yatsui, Email: yatsui@ee.t.u-tokyo.ac.jp

Received 29 June 2015; revised 26 November 2015; accepted 30 November 2015; accepted article preview online 2 December 2015 
an atomic force microscope (AFM) with a 'Sampling Intelligent Scan' mode (Nano Navi, Hitachi-Hitech-Science Corp., Japan).

\section{RESULTS AND DISCUSSION}

Figure 1a and $1 \mathrm{c}$ show the AFM images obtained before etching. From the AFM images, the nano-stripe had a period of $598.4 \pm 14.7 \mathrm{~nm}$ and a pre-etched width, $w_{\mathrm{b}}$, of $438.1 \pm 13.0 \mathrm{~nm}$. In addition, AFM images were taken at the same positions as those shown in Figure 1a and 1c, obtained after 180-min He-Cd laser irradiation with $x$ - (perpendicular to the structure) and $y$ - (parallel to the structure) polarisation, which are shown in Figure $1 \mathrm{~b}$ and $1 \mathrm{~d}$, respectively. The incident light polarisation was controlled with a $\lambda / 2$ waveplate. To evaluate the etching properties, we obtained the cross-sectional profiles in the $x$-direction in the AFM images (Figure 1a-1d). Figure $1 \mathrm{e}-1 \mathrm{~g}$ and $1 \mathrm{~h}-1 \mathrm{j}$ show typical cross-sectional profiles for $x$ - and $y$-polarisation, respectively, taken at different $y$-positions. The dashed and solid curves correspond to images taken before and after etching, respectively, at the same $y$-position, while the black solid curve corresponds to the differences in the pre- and post-etching heights. From the differential height profiles, it is apparent that $x$-polarised etching resulted in uniform etching through the land area; however, the $y$-polarised etching resulted in non-uniform etching. To support this finding, we observed that the surface roughness, $R_{\mathrm{a}}$, at the land area was decreased from 1.14 to $0.60 \mathrm{~nm}$ in the $x$-polarisation case, while $R_{\mathrm{a}}$ was increased from 1.25 to $3.88 \mathrm{~nm}$ in the $y$-polarisation case. Additionally, we compared the etching height, that is, the average differential height through the land width, $w$, which was defined as the full width at $70 \%$ maximum of the pre-etched profile. Figure $1 \mathrm{k}$ shows the obtained etching height as a function of $w$, and indicates that the etching height decreased as $w$ increased.

To determine the etching-property dependence on the polarisation, we calculated the electric and magnetic field distributions using a FDTD method (Fujitsu Poynting for Optics). The cell size was $2 \mathrm{~nm} \times 1 \mathrm{~nm} \times 2 \mathrm{~nm}$ (corresponding to the $x, y$ and $z$ axes, respectively) and the model consisted of $600 \times 1 \times 2000$ cells, with periodic boundary conditions imposed on the $x$ - and $y$-axes and a perfectly matched layer (PML) absorbing boundary condition on the $z$-axis. A light source with a homogenous intensity distribution was set in the $x y$-plane $600 \mathrm{~nm}$ from the top surface of the $\mathrm{ZrO}_{2}$ structure (we used the $\mathrm{ZrO}_{2}$ refractive index of 1.8$)^{11}$. The peak intensity of the incident electric-field was set to $1 \mathrm{~V} \mathrm{~m}^{-1}$. The $\mathrm{ZrO}_{2}$ structure model consisted of a periodic land- and space structure with 50-nm height, $w$-nm land width, and 100-nm spacing (Figure 2a).

Figure $2 \mathrm{~b}-2 \mathrm{i}$ show the polarisation dependence of the electric and magnetic field distributions, with Figure $2 \mathrm{~b}-2 \mathrm{e}$ and $2 \mathrm{f}-2 \mathrm{i}$ displaying the distributions for $w=400$ and $450 \mathrm{~nm}$, respectively. The electric field was localised at the corner of the land structure by the $x$-polarisation, independent of $w$ (Figure $2 \mathrm{~b}$ and $2 \mathrm{f}$ ), while the magnetic field was focused in the vicinity of the land structure centre (Figure $2 \mathrm{~d}$ and $2 \mathrm{~h}$ ). The distributions of both the electric and magnetic fields were dependent on the polarisation. Although the obtained $\mathrm{ZrO}_{2}$ nanostripes were not precisely rectangular, as shown in the AFM images (Figure 1a and 1c), the calculated FDTD results for $\mathrm{ZrO}_{2}$ nano-stripes with tapered structures were almost identical to those for the rectangular structure model (see supplementary information). Thus, the use of a rectangular structure model to obtain the calculated results shown in Figure 2 is considered to be appropriate to explain the experimental results.

The photo-etching performed here used a CW He-Cd laser $(3.81 \mathrm{eV})$, which has lower energy than the $\mathrm{O}_{2}$ photo-dissociation energy
(5.12 eV). Furthermore, the incident laser power was too low to induce a non-linear energy up-conversion. However, many reports have demonstrated that the optical near-field induces energy up-conver$\operatorname{sion}^{14,15}$, allowing the carrier excitation ${ }^{16}$ and the resultant chemical reaction ${ }^{17}$ to use less photon energy than molecule dissociation energy. Furthermore, direct observation of molecular dissociation using the near-field energy up-conversion has been reported ${ }^{18}$. Therefore, the nano-scale etching results shown in this manuscript should originate from the near-field-induced energy up-conversion.

The electric field is localised at the corner of the land structure, which is due to the edge effect, and it may accelerate the etching rate. However, the calculated electric field distributions do not explain the polarisation dependence of the height difference. In contrast, the magnetic field is focused at the centre of the land structure. Figure $3 \mathrm{a}-3 \mathrm{f}$ show a comparison between the magnetic field distributions (the red and blue solid curves indicate $x$ - and $y$ - polarisation, respectively) and height differences (black solid curves). The results indicate that the height-difference profiles are in good qualitative agreement with the magnetic field distributions. That is, the etching profiles can be regarded as being governed by the magnetic field distributions, rather than the electric field distributions.

Additionally, as the nano-scale etching was realised by the localised magnetic field, the peak value of the magnetic field intensities was plotted as a function of the $w$ (see Figure $3 \mathrm{~g}$ ), leading to decreased magnetic field intensity with increasing $w$. The dependence of the magnetic field intensity on $w$ is similar to the experimental results for the etching height shown in Figure 1k. Taking the variations in the magnetic field distribution into consideration (see Figure 2d, 2e, $2 \mathrm{~h}$, and $2 \mathrm{i}$ ), the increase in the etching rate as $w$ decreases may contribute to higher magnetic-field concentration, with the land structure constructing the magnetic-field interference.

To determine the magnetic field distributions shown in Figure 2, we observed the electric and magnetic field components. Figure $4 \mathrm{a}$ and $4 \mathrm{~b}$ show the $x$ - and $y$-components of the magnetic field for $x$ - and $y$ polarisation, respectively, where the $z$-components were negligible for both types of polarisation. From these results, it is apparent that the magnetic field distributions due to $x$ - and $y$-polarisation were determined by the $y$ - (red solid squares in Figure 4a) and $x$ - (blue solid circles in Figure 4b) components of the magnetic field, respectively. As is observed in Figure $2 b(2 \mathrm{f})$ and $2 \mathrm{c}(2 \mathrm{~g})$, the electric field is parallel to the incident light polarisation. The electric fields are generated as shown in Figure 4c and 4d, while the generated magnetic-field distributions (black solid diamonds in Figure $4 \mathrm{a}$ and $4 \mathrm{~b}$ ) are perpendicular to the incident light polarisation (Figure $4 \mathrm{c}$ and $4 \mathrm{~d}$ ). To evaluate the strength of the generated magnetic field, we compared the magnetic field intensity obtained with nano-stripe structures with that in free space, i.e., in the absence of nano-stripe structures (dashed black lines in Figure $4 \mathrm{a}$ and $4 \mathrm{~b}$ ). The peak magnetic field intensity generated using nano-stripe structures was twice that without them. As the polarisation dependence results for the nano-scale etching are qualitatively explained by the magnetic field distributions, rather than the electric field distributions, the nano-scale etching was governed by the localised magnetic field accompanying the energy up-conversion.

As described, the near-field etching originated from the radical oxygen species, which was dissociated from the $\mathrm{O}_{2}$ using the near-field energy up-conversion. The localisation of the optical near-field induces not only an electric dipole transition, but also an optically inactive transition including the magnetic dipole and magnetic quadrupole transitions, along with other higher-order transitions. In other words, the optical near-field allows activation of the optically inactive 

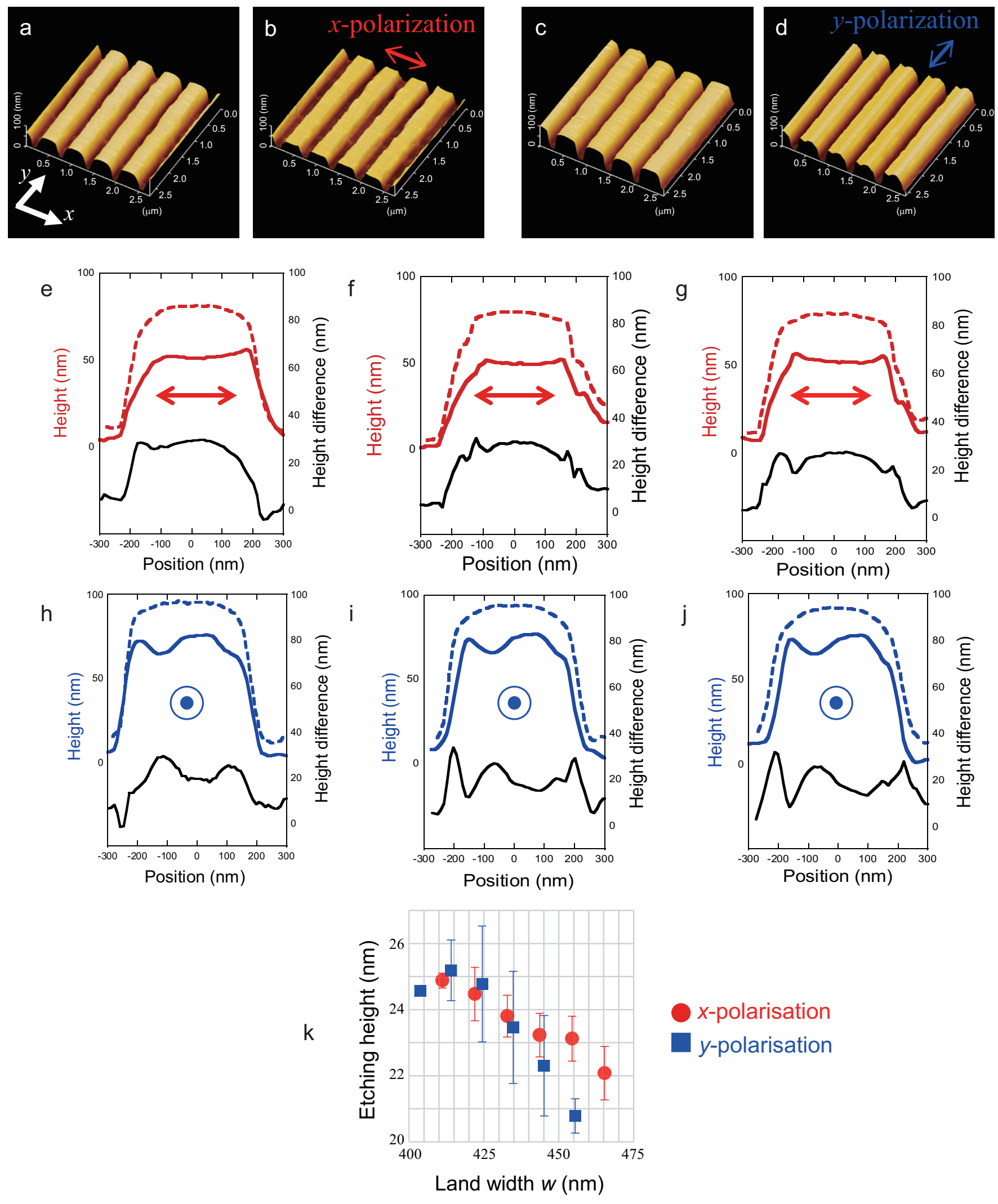

Figure 1 Polarisation-dependent etching. (a) and (c) are AFM images taken before etching. (b) and (d) are AFM images after 3 h. Perpendicular ( $x$-polarised) and parallel ( $y$-polarised) samples are shown in (b) and (d), respectively. Images (a) and (b), and (c) and (d), were obtained at the same respective positions. (e-g) Crosssectional profiles of $x$-polarised samples. Red dashed line: pre-etched sample in (a), red solid line: etched sample in (b), black solid line: difference in height before and after etching. The pre-etched widths, $w_{\mathrm{b}}$, were (e) 411.17, (f) 432.81 , and (g) $454.45 \mathrm{~nm}$. (h-j) Cross-sectional profiles of $y$-polarised samples. Blue dashed line: preetched sample in (c), blue solid line: etched sample in (d), black solid line: difference in height before and after etching. $w_{\mathrm{b}}$ were (h) 414.06 , (i) 424.41 , and (j) $445.11 \mathrm{~nm}$. (k) Etching height as a function of $w$. 


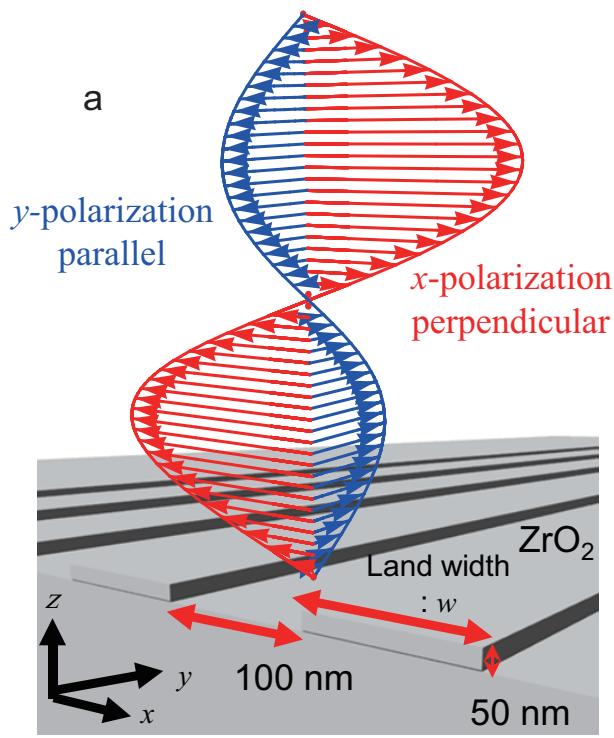

b
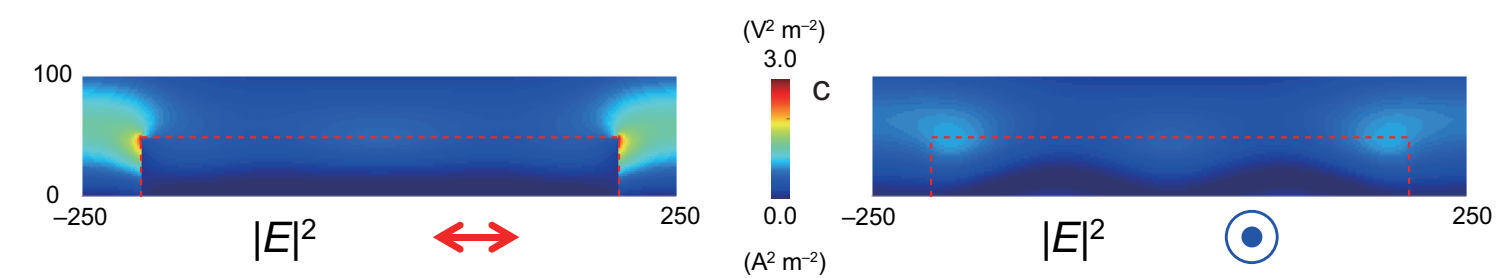

d

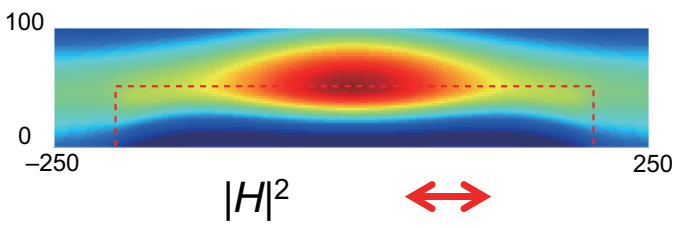

$1.5 \times 10^{-5}$
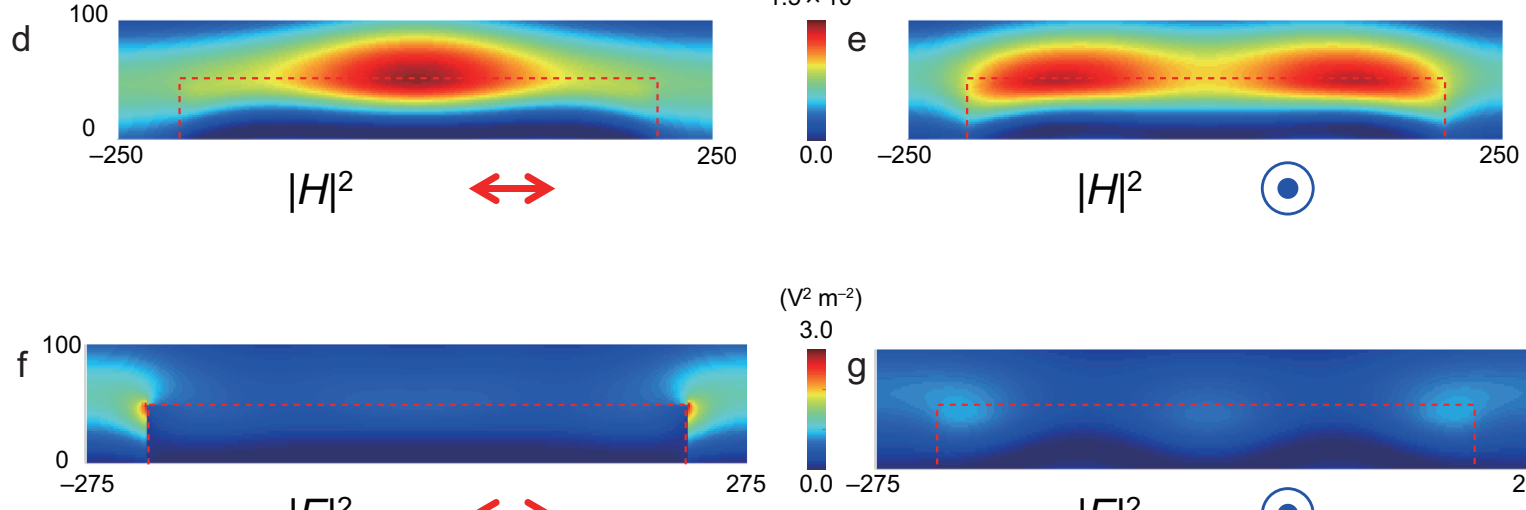

$\left(\mathrm{V}^{2} \mathrm{~m}^{-2}\right)$

3.0

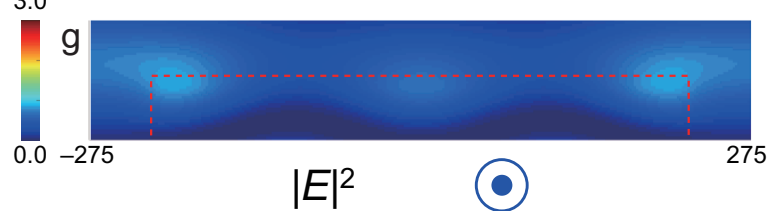

$\left(\mathrm{A}^{2} \mathrm{~m}^{-2}\right)$

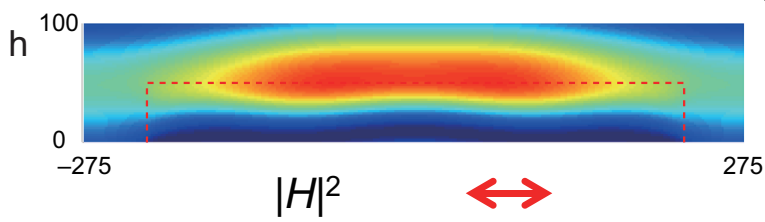

$1.5 \times 10^{-5}$
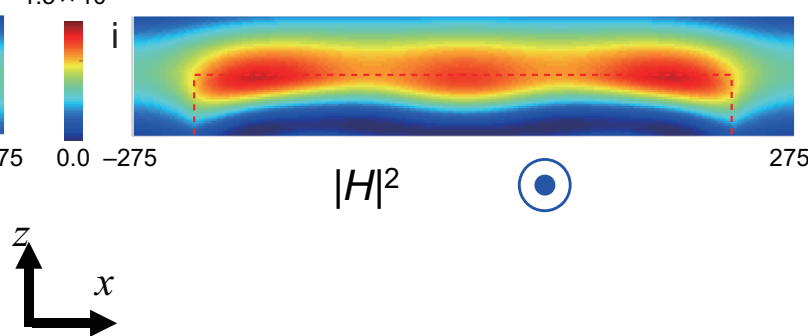

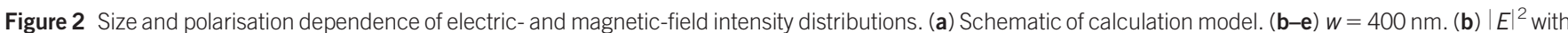

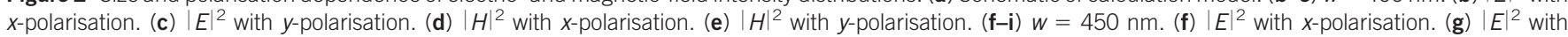
$y$-polarisation. (h) $\mid H^{2}$ with $x$-polarisation. (i) $|H|^{2}$ with $y$-polarisation. The red dashed line corresponds to the $\mathrm{ZrO}_{2}$ land $\mathrm{profiles}$ 

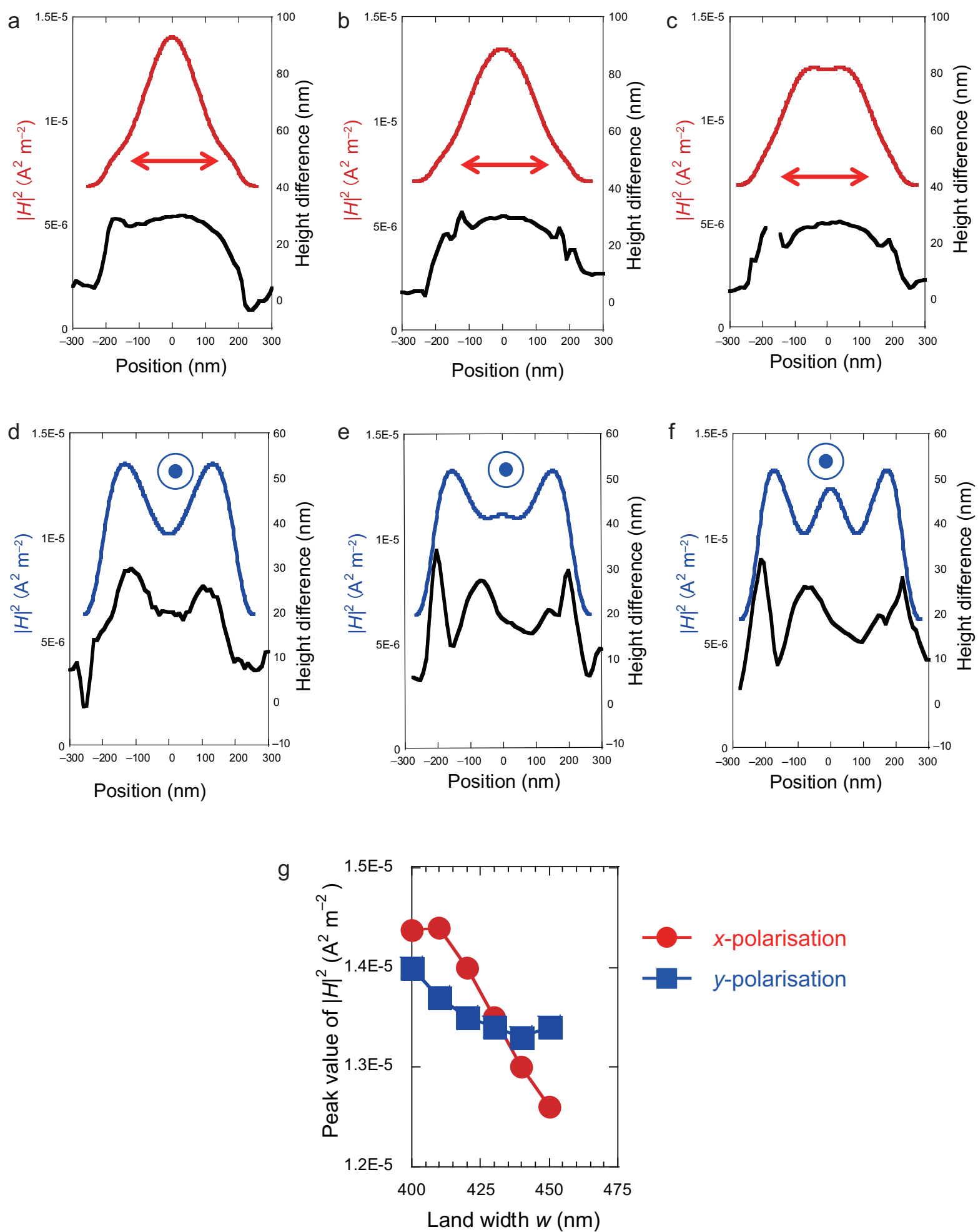

Figure 3 Comparison between pre- and post-etching heights. In (a-f), the red/blue and black solid lines correspond to the calculated $|H|^{2}$ and the change in etching height, respectively. Further, the red and blue solid lines correspond to $x$ - and $y$-polarisation, respectively. The calculated wand $w_{\mathrm{b}}$ with $x$-polarisation are (a) 410 and 411.17, (b) 430 and 432.81, and (c) 450 and $454.45 \mathrm{~nm}$, respectively. The calculated wand the pre-etching widths, $w_{\mathrm{b}}$, with $y$-polarisation are (d) 410 and 414.06 , (e) 430 and 424.41 , and (f) 450 and 445.11 nm, respectively. (g) Peak values of calculated magnetic-field intensity as a function of $w$.

intermediate states ${ }^{19,20}$. As for the $\mathrm{O}_{2}$ potential energy curves, it has been reported that $\mathrm{O}_{2}$ has strong intermediate states such as $c^{1} \Sigma_{u}^{-}$ (transition energy from the ground state $E=4.05 \mathrm{eV}$ ) and $A^{3} \Sigma_{u}^{+}$
$(E=4.33 \mathrm{eV})^{21}$, which have transition energies from the ground state $\left(X^{3} \Sigma_{g}^{-}\right)$that are higher than the incident photon energy $\left(E_{1}=3.81 \mathrm{eV}\right)$. Transitions from the ground states, that is, the magnetic quadrupole 
a

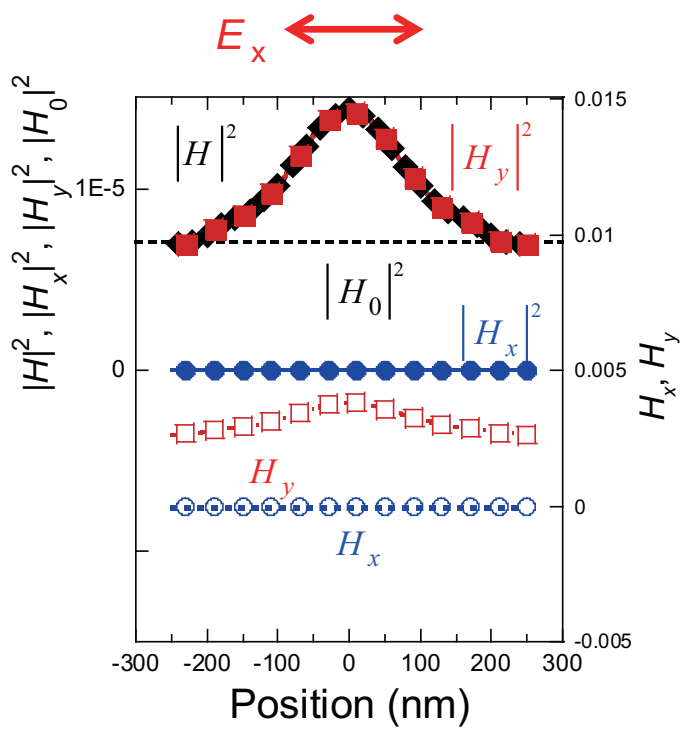

b

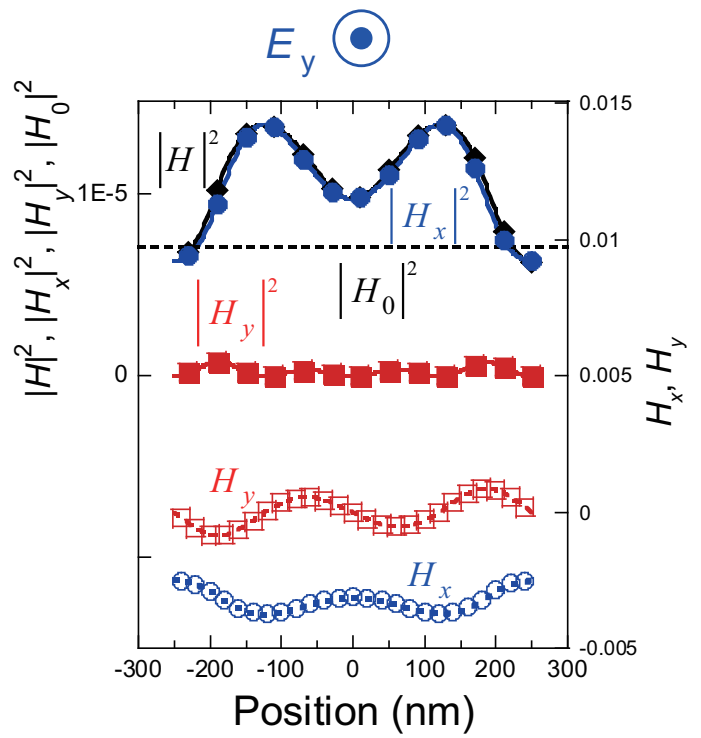

C

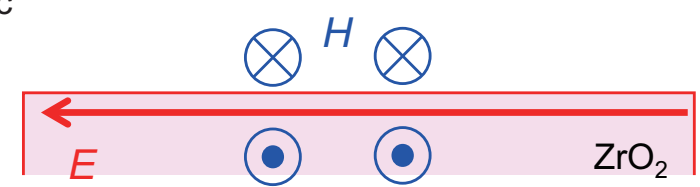

d

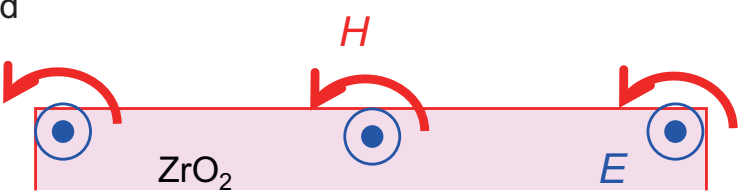

Figure 4 Magnetic field distributions depending on incident light polarisations. (a) $x$-polarisation, (b) $y$-polarisation. $\left|H_{0}\right|$ indicates the magnetic field intensity in the absence of nano-stripe structures. Schematic of magnetic-field generation for (c) $x$ - and (d) $y$-polarisation. The calculated $w$ is $400 \mathrm{~nm}$.

transitions $\left(c^{1} \Sigma_{u}^{-22}\right.$ and $\left.A^{3} \Sigma_{u}^{+21}\right)$, are all optically forbidden. Note that it is the second transition step from the intermediate to the excited state that is optically forbidden, i.e., the magnetic dipole transition $\left(c^{1} \Sigma_{u}^{-} \rightarrow B^{3} \Sigma_{u}^{-}\right.$and $\left.A^{3} \Sigma_{u}^{+} \rightarrow B^{3} \Sigma_{u}^{-}\right)$. The strength of the magnetic dipole transition depends on the intensity of the oscillating magnetic field. As the calculated magnetic field shown in Figure 2 is an oscillating field, the agreement of the height-difference profiles with the calculated magnetic field distributions shown in Figure3 supports the finding that the localised magnetic field induces the nano-scale etching. This postulate is also supported by reports on the generation of the magnetic dipole moment by the near-field excitation, in which the magnetic dipole moment generation originates from the second harmonic electric field in the near-field ${ }^{23}$. Therefore, the localised magnetic field accompanying the energy up-conversion causes the chemical reaction via the optically inactive intermediate state.

\section{CONCLUSIONS}

Through the nano-scale etching experiments described above, we discovered evidence of the strong magnetic-field interaction with the material excitation. Since conventional nano-scale fabrication using the near-field is designed considering the electric field only ${ }^{7,24}$, the incorporation of the magnetic field will provide an additional degree of freedom during the fabrication of advanced optical and/or electrical device structures on the nano-scale. Furthermore, the optically controlled magnetic-field interaction with material excitation on the nano-scale will allow new spin manipulation in space and time to be realised, in the absence of an external magnetic field ${ }^{25}$.

\section{ACKNOWLEDGMENT}

The authors wish to express special thanks to Prof. Motoichi Ohtsu (University of Tokyo) and Prof. Itsuki Bannno (Yamanashi University) for active support and discussion. We thank Mrs. Etsuko Ota (University of Tokyo) for the AFM measurements. This work was partially supported by a MEXT Grant-in-Aid for Scientific Research (B) (No. 26286022, 25288012), a MEXT Grant-in-Aid for Exploratory Research Program (No. 26630122), a MEXT Grant-in-Aid for Scientific Research on Innovative Areas (No. 15H00866), a MEXT Nanotechnology Platform (No.12024046), the JSPS Core-to-Core Program (A. Advanced Research Networks), the Yazaki Memorial Foundation, the Research Foundation for Opto-Science and Technology, and the RIKEN AICS through the HPCI System Research project (ID: hp150218).

1 Dürig U, Pohl DW, Rohner F. Near-field optical-scanning microscopy. J Appl Phys 1986; 59: 3318-3327.

2 Betzig E, Chichester RJ. Single molecules observed by near-field scanning optical microscopy. Science 1993; 262: 1422-1425.

3 Matsuda K, Saiki T, Nomura S, Mihara M, Aoyagi Y et al. Near-field optical mapping of exciton wave functions in a GaAs quantum dot. Phys Rev Lett 2003; 91: 177401.

4 Yano T, Verma P, Saito Y, Ichimura T, Kawata S. Pressure-assisted tip-enhanced Raman imaging at a resolution of a few nanometres. Nat Photonics 2009; 3 473-477.

5 Shalaev VM. Optical negative-index metamaterials. Nat Photonics 2007; 1: 41-48.

6 Veselago VG. The electrodynamics of substances with simultaneously negative values of $\varepsilon$ and $\mu$. Usp Fiz Nauk 1964; 92: 517-526.

7 Fang $\mathrm{N}$, Lee $\mathrm{H}$, Sun C, Zhang X. Sub diffraction-limited optical imaging with a silver superlens. Science 2005; 308: 534-537.

8 Ergin T, Stenger N, Brenner P, Pendry JB, Wegener M. Three-dimensional invisibility cloak at optical wavelengths. Science 2010; 328: 337-339.

9 Sheng C, Liu H, Wang Y, Zhu SN, Genov DA. Trapping light by mimicking gravitational lensing. Nat Photonics 2013; 7: 902-906.

10 Ridaoui H, Wieder F, Ponche A, Soppera O. Direct ArF laser photopatterning of metal oxide nanostructures prepared by the sol-gel route. Nanotechnology 2010; 21 065303.

11 Stehlin F, Wieder F, Spangenberg A, Le Meins J-M, Soppera O. Room-temperature preparation of metal-oxide nanostructures by DUV lithography from metal-oxo clusters. J Mater Chem C 2014; 2: 277-285.

12 Yatsui T, Nomura W, Stehlin F, Soppera O, Naruse M et al. Challenges in realizing ultraflat materials surfaces. Beilstein J Nanotechnol 2013; 4: 875-885.

13 Keilin D, Hartree EF. Absorption spectrum of oxygen. Nature 1950; 165: 543-544. 
14 Yatsui T, Sangu S, Kobayashi K, Kawazoe T, Ohtsu M et al. Nanophotonic energy up conversion using ZnO nanorod double-quantum-well structures. Appl Phys Lett2009; 94: 083113.

15 Yamaguchi M, Nobusada K, Kawazoe T, Yatsui T. Two-photon absorption induced by electric field gradient of optical near-field and its application to photolithography. Appl Phys Lett 2015; 106: 191103.

16 Kawazoe T, Fujiwara H, Kobayashi K, Ohtsu M. Visible light emission from dye molecular grains via infrared excitation based on the nonadiabatic transition induced by the optical near field. IEEE J Select Top Quant Electron 2009; 15: 1380-1386.

17 Kawazoe T, Kobayashi K, Takubo S, Ohtsu M. Nonadiabatic photodissociation process using an optical near field. J Chem Phys 2005; 122: 024715.

18 Tanjeem N, Kawazoe T, Yatsui T. $\mathrm{CO}_{2}$ phonon mode renormalization using phononassisted energy up-conversion. Sci Rep 2013; 3: 3341.

19 Feng TH, Zhou Y, Liu DH, Li J. Controlling magnetic dipole transition with magnetic plasmonic structures. Opt Lett 2011; 36: 2369-2371.

20 Hein SM, Giessen H. Tailoring magnetic dipole emission with plasmonic split-ring resonators. Phys Rev Lett 2013; 111: 026803.

21 Krupenie PH. The spectrum of molecular oxygen. J Phys Chem Ref Data 1972; 1: 423-534.
22 Mizushima M. $\Delta \mathrm{S}= \pm 1$ Magnetic quadrupole radiative transitions in atoms and molecules. J Phys Soc Jpn 1966; 21: 2335-2344.

23 Yamaguchi M, Nobusada K, Yatsui T. Nonlinear optical response induced by a secondharmonic electric-field component concomitant with optical near-field excitation. Phys Rev A 2015; 92: 043809.

24 Challener WA, Peng CB, Itagi AV, Karns D, Peng W et al. Heat-assisted magnetic recording by a near-field transducer with efficient optical energy transfer. Nat Photonics 2009; 3: 220-224.

25 Kato Y, Myers RC, Gossard AC, Awschalom DD. Coherent spin manipulation without magnetic fields in strained semiconductors. Nature 2004; 427: 50-53.

(c) (i) This work is licensed under a Creative Commons Attribution 4.0 Unported License. The images or other third party material in this article are included in the article's Creative Commons license, unless indicated otherwise in the credit line; if the material is not included under the Creative Commons license, users will need to obtain permission from the license holder to reproduce the material. To view a copy of this license, visit http://creativecommons.org/licenses/by/4.0/

Supplementary information for this article can be found on the Light: Science \& Applications' website (http://www.nature.com/lsa/). 